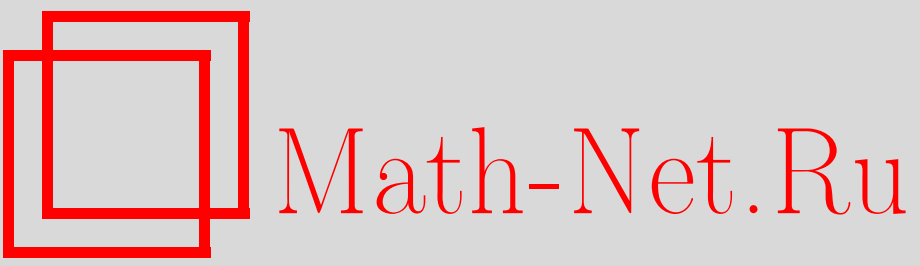

Д. Е. Пелиновский, К. Салем, Асимптотики нового собственного значения в линейных задачах без порога, ТМФ, 2000, том 122, номер 1, 118-127

DOI: https://doi.org/10.4213/tmf559

Использование Общероссийского математического портала Math-Net.Ru подразумевает, что вы прочитали и согласны с пользовательским соглашением

http://www.mathnet.ru/rus/agreement

Параметры загрузки:

IP: 3.80 .181 .102

26 апреля 2023 г., 17:05:09 
ТЕОРЕТИЧЕСКАЯ

И МАТЕМАТИЧЕСКАЯ

ФИЗИКА

Том 122, № 1

январь, 2000

(C) 2000 г

Д. Е. Пелиновский* , К. Салем*

\section{АСИМПТОТИКИ НОВОГО СОБСТВЕННОГО ЗНАЧЕНИЯ В ЛИНЕЙНЫХ ЗАДАЧАХ БЕЗ ПОРОГА}

Исследуется критерий появления нового собственного значения в линейной спектральной задаче, соответствующей промежуточному уравнению длинных волн. Методом фурье-разложения вычисляется асимптотика нового собственного значения в пределе малого потенциала. Результаты сравниваются с аналогичными результатами для оператора Шредингера с радиально-симметричным потенциалом.

\section{1. ВВЕДЕНИЕ}

Хорошо известно, что у оператора Шредингера $\mathcal{L}=-\nabla_{d}^{2}+\epsilon v(r)$ при малой константе связи $\epsilon \ll 1$ может сушествовать слабо связанное состояние $\mathcal{L} \Phi_{b}=\lambda_{b} \Phi_{b}$, где $\lambda=\lambda_{b}(\epsilon)<0$ [1]. Оно заведомо существует в одномерном и двумерном случаях, если полная масса

$$
M \propto \int_{0}^{\infty} r^{d-1} v(r) d r
$$

потенциала $v=v(r)$ отрицательна [2]. Методами операторного анализа были изучены аналитические свойства функции $\lambda=\lambda_{b}(\epsilon)[3]$; позднее эти результаты были обобщены на случай $\lambda=\lambda_{b}\left(\epsilon-\epsilon_{0}\right)$, где $\epsilon_{0}-$ пороговое значение [4]. Недавно с помощью интегрального представления Апенко получил [5] асимптотики нового собственного значения для случая $d \geqslant 2$.

Развитый для оператора Шредингера анализ был ограничен самосопряженными дифференциальными операторами второго порядка. В связи с теориями солитонов изучались также и другие линейные задачи на собственные значения, например задачи, связанные с матричными несамосопряженными дифференциальными операторами [6, 7], или дифференциальные спектральные задачи Римана-Гильберта [8] .

В настоящей работе мы предлагаем простой асимптотический подход, основанный на фурье-разложении собственных функций слабо связанных состояний, и находим асимптотики новых собственных значений в некоторых линейных задачах. Используемый при этом метод известен в квантовой механике как борновское приближение для амплитуды волны, рассеянной на малом потенциале (см. [2, гл. XVII, п. 130]). Метод применяется для описания рождения солитонов в системе, описываемой промежуточным

\footnotetext{
${ }^{*}$ Department of Mathematics, University of Toronto, Toronto, Ontario, Canada
} 
уравнением длинных волн

$$
u_{t}+\delta^{-1} u_{x}+2 \epsilon u u_{x}+T\left(u_{x x}\right)=0
$$

где $\delta$ - параметр, $T(u)$ - сингулярный интегродифференциальный оператор

$$
T(u)=\frac{1}{2 \delta} \mathrm{v} \cdot \mathrm{p} \cdot \int_{-\infty}^{\infty} \operatorname{cth}\left[\frac{\pi(z-x)}{2 \delta}\right] u(z) d z
$$

и $\epsilon \ll 1$. Этому уравнению соответствует следующая линейная задача на собственные значения [9]:

$$
i w_{x}^{+}+\left(q(k)+\frac{1}{2 \delta}\right)\left(w^{+}-w^{-}\right)+\epsilon u(x) w^{+}=0
$$

где

$$
q(k)=k \frac{\exp (2 k \delta)}{\operatorname{sh}(2 k \delta)}-\frac{1}{2 \delta},
$$

$k$ - спектральный параметр, а $w^{ \pm}=w(x \mp i \delta)$. В работе [10] изучалось рождение солитонов большим начальным импульсом $(\epsilon \gg 1)$. В данной работе анализируется противоположный случай. Показано, что при $k=i \kappa(\epsilon)$ и $\epsilon \rightarrow 0$ слабо связанное состояние всегда существует, если масса

$$
M=\int_{-\infty}^{\infty} u(x) d x
$$

строго положительна. Это связанное состояние отвечает солитону промежуточного уравнения длинных волн (1), генерируемому малым начальным импульсом.

В пределе $\delta \rightarrow 0$ справедливо следуюшее разложение:

$$
T\left(u_{x}\right)+\delta^{-1} u=\frac{\pi}{4 \delta^{2}} \mathrm{v} \cdot \mathrm{p} \cdot \int_{-\infty}^{\infty} \frac{u(z)-u(x)}{\operatorname{sh}^{2}\left[\frac{\pi(z-x)}{2 \delta}\right]} d z=\frac{\delta}{\pi^{2}} u_{x x} \int_{-\infty}^{\infty} \frac{z^{2} d z}{\operatorname{sh}^{2}(z)}+O\left(\delta^{3}\right),
$$

где последний интеграл равен $\pi^{2} / 3$ [11]. Тогда промежуточное уравнение длинных волн (1) сводится к уравнению Кортевега-де Фриза (КдФ)

$$
3 u_{t}+6 \epsilon u u_{x}+\delta u_{x x x}=0
$$

которому соответствует одномерный оператор Шредингера

$$
\delta\left(w_{x x}-2 i k w_{x}\right)+\epsilon u(x) w=0 .
$$

В этом пределе новое собственное значение $k=i \kappa(\epsilon)$ имеет асимптотику вида

$$
\kappa(\epsilon)=\frac{\epsilon}{2 \delta} M+O\left(\epsilon^{2}\right)
$$

где $M>0$. Оказывается, что новое собственное значение линейной задачи $(2)$ при конечном $\delta$ имеет асимптотику такого же вида, т.е. $\kappa(\epsilon) \sim O(\epsilon)$. В пределе $\delta \rightarrow \infty$, когда промежуточное уравнение длинных волн переходит в уравнение 
Бенджамина-Оно (БО) [9], величина $\kappa(\epsilon)$ как функция $\epsilon$ экспоненциально мала, т.е. $\kappa \sim O\left(\exp \left(-2 \pi \epsilon^{-1} M^{-1}\right)\right)$, где $M>0$ [8]. Таким образом, при любом значении $\delta$ в спектральной задаче (2) не сушествует порогового значения амплитуды $\epsilon$ потенциала, при котором появляется новое связанное состояние. Это означает, что для промежуточного уравнения длинных волн (1), так же как и для его предельных случаев, уравнений КдФ и БО, любой начальный импульс с положительной массой $M$ с течением времени преврашается в солитон.

Этот результат сравнивается с результатом анализа $d$-мерного оператора Шредингера с радиально-симметричным потенциалом

$$
\phi_{r r}+\frac{d-1}{r} \phi_{r}+k^{2} \phi+\epsilon u(r) \phi=0, \quad r \geqslant 0,
$$

где $k$ - спектральный параметр. Мы исследуем разложение Фурье-Бесселя слабо связанного состояния и показываем, что в спектральной задаче (7) при $0<d \leqslant 2$ не сушествует порога по $\epsilon$ для возникновения нового связанного состояния. При $0<d<2$ для нового собственного значения $k=i \kappa(\epsilon)$ получена асимптотика вида $\kappa \sim O\left(\epsilon^{1 /(d-2)}\right)$. Таким образом, в линейной задаче (7) собственное значение $k=i \kappa(\epsilon)$ не является, вообше говоря, аналитической функцией $\epsilon$, в то время как в линейной задаче (2) оно является аналитической функцией при любом конечном значении $\delta$. При $d=1$ результат сводится к зависимости $\kappa \sim O(\epsilon)$. При $d \rightarrow 2^{-}$функция $\kappa(\epsilon)$ становится экспоненциально малой по $\epsilon$, т.е. $\kappa \sim O\left(\exp \left(-2 \pi \epsilon^{-1} M^{-1}\right)\right)$, где $M>0$ - полная масса потенциала. При $d=2$ наш подход позволяет вычислить предэкспоненциальный фактор. Последний результат дополняет недавний анализ Апенко [5], который рассматривал задачу (7) при $d>2$, когда новое собственное значение возникает при $\epsilon>\epsilon_{0}$, где $\epsilon_{0}-$ пороговое значение. Он также вычислил предэкспоненциальный фактор в пределе $d \rightarrow 2^{+}$.

\section{2. ПРОМЕЖУТОЧНОЕ УРАВНЕНИЕ ДЛИННЫХ ВОЛН}

Мы рассматриваем линейную задачу (2) при конечном $\delta$ как дифференциально-разностную задачу в комплексной плоскости $x$. При $\epsilon=0$ имеются два решения: $M^{ \pm}(x, k)=1$ и $N^{ \pm}(x, k)=e^{2 i k(x \mp i \delta)}$. Мы строим решение уравнения (2) при $q=$ $q(i \kappa(\epsilon))$ и $0<\epsilon \ll 1$ в виде разложения Фурье по собственным функциям $N^{ \pm}(x, k)$ :

$$
w^{ \pm}(x)=\int_{-\infty+i 0}^{\infty+i 0} \alpha(k) e^{2 i k(x \mp i \delta)} d k,
$$

где $\alpha(k)$ - коэффициенты Фурье, а сдвиг $k \rightarrow k+i 0$ пределов интегрирования гарантирует, что собственные функции $w^{ \pm}(x)$ обрашаются в нуль при $x \rightarrow+\infty$ (в противном случае связанное состояние не будет локализованным). При подстановке (8) в уравнение (2) последнее сводится к интегральному уравнению

$$
\beta(k)=\frac{\epsilon}{4 \pi \delta} \int_{-\infty}^{\infty} \frac{\left(1+2 \delta q\left(k^{\prime}\right)\right) K\left(k, k^{\prime}\right) \beta\left(k^{\prime}\right) d k^{\prime}}{\left(k^{\prime}+i 0\right)\left(q\left(k^{\prime}\right)-q(i \kappa)\right)},
$$

где

$$
\beta(k)=\frac{k e^{2 k \delta}(q(k)-q(i \kappa))}{\left(q(k)+(2 \delta)^{-1}\right)} \alpha(k)
$$




$$
K\left(k, k^{\prime}\right)=\int_{-\infty}^{\infty} u(x) e^{2 i\left(k^{\prime}-k\right) x} d x .
$$

Интегральное уравнение (9) сингулярно при $k^{\prime} \rightarrow 0$, если $q(i \kappa) \rightarrow 0$, т.к. асимптотическое разложение для $q(k)$ при $k \rightarrow 0$ имеет вид

$$
q(k)=k+\frac{2}{3} k^{2} \delta+O\left(k^{3} \delta^{2}\right)
$$

Чтобы получить правильный предел для (9) при $\epsilon \rightarrow 0$, мы вычисляем полюсные вклады при $k^{\prime}=-i 0$ и $k^{\prime}=i \kappa$ и переписываем уравнение (9) в эквивалентной форме

$$
\begin{aligned}
\beta(k)= & \frac{\epsilon(k, 0) \beta(0)}{4 \delta \kappa}\left[\frac{1+2 \delta q(i \kappa)}{q^{\prime}(i \kappa)}+\frac{i \kappa}{q(i \kappa)}\right]+\frac{\epsilon}{4 \pi \delta} \int_{-\infty}^{\infty} \frac{d k^{\prime}}{\left(k^{\prime}+i 0\right)\left(k^{\prime}-i \kappa\right)} \times \\
& \times\left[\frac{\left(1+2 \delta q\left(k^{\prime}\right)\right)\left(k^{\prime}-i \kappa\right)}{q\left(k^{\prime}\right)-q(i \kappa)} K\left(k, k^{\prime}\right) \beta\left(k^{\prime}\right)-\right. \\
& \left.-\left(\frac{i \kappa}{q(i \kappa)}+\frac{k^{\prime}(1+2 \delta q(i \kappa))}{i \kappa q^{\prime}(i \kappa)}-\frac{k^{\prime}}{q(i \kappa)}\right) K(k, 0) \beta(0)\right],
\end{aligned}
$$

где $q^{\prime}(k)=d q(k) / d k$ и принято, что $\kappa(\epsilon)>0$. Из первого члена уравнения (11) ясно, что асимптотический баланс достигается при $\kappa \sim O(\epsilon)$. Поэтому мы полагаем, что справедливо разложение $\kappa=\epsilon \kappa_{1}+\epsilon^{2} \kappa_{2}+O\left(\epsilon^{3}\right)$, так что в пределе $\epsilon \rightarrow 0$ уравнение (11) принимает вид

$$
\begin{aligned}
\beta(k)= & \frac{K(k, 0) \beta(0)}{2 \delta \kappa_{1}}\left(1-\epsilon \frac{\kappa_{2}}{\kappa_{1}}\right)+ \\
& +\frac{\epsilon}{4 \pi \delta} \mathrm{v} \cdot \mathrm{p} \cdot \int_{-\infty}^{\infty} \frac{d k^{\prime}}{k^{\prime 2}}\left[\frac{k^{\prime}\left(1+2 \delta q\left(k^{\prime}\right)\right)}{q\left(k^{\prime}\right)} K\left(k, k^{\prime}\right) \beta\left(k^{\prime}\right)-K(k, 0) \beta(0)\right]+O\left(\epsilon^{2}\right) .
\end{aligned}
$$

Из условия совместности уравнения (12) при $k=0$ следует асимптотическое разложение для $\beta(k)$

$$
\begin{aligned}
\kappa_{1} & =\frac{1}{2 \delta} M, \\
\kappa_{2} & =\frac{1}{8 \pi \delta^{2}} \iint_{-\infty}^{\infty} u(x) u(y) Q(x-y) d x d y,
\end{aligned}
$$

где $M$ определяется соотношением (4), а

$$
Q(z)=\text { v.p. } \int_{-\infty}^{\infty} \frac{d k}{k^{2}}\left[\frac{k}{q(k)} e^{2 i k z}-1\right] .
$$

Формула для $\kappa(\epsilon)$ справедлива при условии, что $\kappa_{1}>0$ (т.е. $M>0$ ) и что величина $\kappa_{2}$ конечна (необходимое условие сходимости разложения). В пределе $\delta \rightarrow 0$ с помошью равенства

$$
\frac{1}{2 \pi} \mathrm{v} \cdot \mathrm{p} \cdot \int_{-\infty}^{\infty} \frac{d k}{k^{2}}\left(e^{2 i k z}-1\right)=-|z|
$$


соотношение (14) упрошается до

$$
\kappa_{2}=-\frac{1}{4 \delta^{2}} \iint_{-\infty}^{\infty} u(x) u(y)|x-y| d x d y
$$

Последнее выражение описывает поправку к новому собственному значению одномерного опратора Шредингера, т.е. уравнения (6) (см. также [3]). С помощью теоремы о вычетах функцию $Q(z)$ в (14) при конечном $\delta$ можно формально представить в виде суммы ведущего члена $-2 \pi|z|$ и вкладов от бесконечного числа полюсов, отвечающих нулям $q(k)$ при $\operatorname{Im}(k)>0$ [9]. Соответственно асимптотика нового собственного значения промежуточного уравнения длинных волн имеет вид

$$
\kappa(\epsilon)=\frac{\epsilon M}{2 \delta}[1+O(\epsilon)]
$$

где $M>0$. В пределе $\delta \rightarrow \infty$, когда промежуточное уравнение длинных волн переходит в уравнение БО, коэффициенты $\kappa_{1}, \kappa_{2}$, так же как и высшие члены разложения, обращаются в нуль. В этом случае собственное значение $\kappa(\epsilon)$ спектральной задачи для промежуточного уравнения длинных волн (2) переходит в собственное значение уравнения БО нетривиальным образом. Этот переход выходит за рамки асимптотического разложения по степеням $\epsilon$.

Чтобы воспроизвести результаты решения спектральной задачи для уравнения БО, заметим, что собственное значение $q(i \kappa)$, определяемое формулой $(3)$, не является хорошо определенной величиной в пределе $\delta \rightarrow \infty$, если не потребовать $[9,10]$, чтобы

$$
\kappa=\frac{\pi}{2 \delta}\left(1+\frac{1}{4 \delta k_{0}}\right),
$$

где $k_{0}$ - параметр. Тогда получаются следующие разложения $q(i \kappa)$ и $q(k)$ при больших $\delta$ :

$$
\begin{aligned}
q(i \kappa) & =2 k_{0}+O\left(\delta^{-1}\right), \\
q(k) & =2 k \Theta(k)+O\left(\delta^{-1}\right),
\end{aligned}
$$

где $\Theta(k)$ - функция Хевисайда: $\Theta(k)=1$ при $k>0$ и $\Theta(k)=0$ при $k<0$. Действительньй отрицательный параметр $k_{0}(\epsilon)$ определяет собственное значение уравнения БО, которое экспоненциально мало по $\epsilon$ [8]. С учетом формул (17) и (18) интегральное уравнение (9) в пределе $\delta \rightarrow \infty$ принимает вид

$$
\beta(k)=\frac{\epsilon}{2 \pi} \int_{0}^{\infty} \frac{K\left(k, k^{\prime}\right) \beta\left(k^{\prime}\right) d k^{\prime}}{\left(k^{\prime}-k_{0}\right)}, \quad k \geqslant 0 .
$$

Интеграл в правой части (19) сингулярен при $k_{0} \rightarrow 0$ из-за наличия точки ветвления при $k^{\prime} \rightarrow 0$. Полагая $k_{0}=-p(\epsilon)$, представим уравнение $(19)$ в виде

$$
\begin{aligned}
\beta(k)=-\frac{\epsilon}{2 \pi} & \ln \left(\frac{p}{1+p}\right) K(k, 0) \beta(0)+ \\
& +\frac{\epsilon}{2 \pi}\left[\int_{0}^{1} \frac{K\left(k, k^{\prime}\right) \beta\left(k^{\prime}\right)-K(k, 0) \beta(0)}{\left(k^{\prime}+p\right)} d k^{\prime}+\int_{1}^{\infty} \frac{K\left(k, k^{\prime}\right) \beta\left(k^{\prime}\right)}{\left(k^{\prime}+p\right)} d k^{\prime}\right] .
\end{aligned}
$$


Условие асимптотического баланса удовлетворяется, если $\ln p \sim O\left(\epsilon^{-1}\right)$. Следовательно, можно написать $\ln p=-\epsilon^{-1} p_{-1}+p_{0}+O(\epsilon)$, где $p_{-1}>0$, и рассмотреть в уравнении (20) предел $p \rightarrow 0$. Полагая $k=0$, находим явные выражения:

$$
\begin{aligned}
p_{-1} & =\frac{2 \pi}{M}, \\
p_{0} & =-\gamma-\ln 2-\frac{1}{M^{2}} \iint_{-\infty}^{\infty} u(x) u(y) \ln |x-y| d x d y,
\end{aligned}
$$

где $\gamma \approx 0.577$ - константа Эйлера; при вычислении интегралов мы воспользовались формулами (3.721) и (3.782) из [11]:

$$
\int_{0}^{1} \frac{e^{2 i k z}-1}{k} d k+\int_{1}^{\infty} \frac{e^{2 i k z}}{k} d k=-\gamma-\ln 2-\ln |z|+\frac{i \pi}{2} \operatorname{sign}(z) .
$$

Таким образом, асимптотика собственного значения уравнения БО имеет вид

$$
k_{0}(\epsilon)=-c \exp \left(-2 \pi \epsilon^{-1} M^{-1}\right)[1+O(\epsilon)],
$$

где $c=\exp \left(p_{0}\right)$ и $M>0$. Из (16) следует, что при больших, но конечных $\delta$, собственное значение $\kappa(\epsilon)$ экспоненциально велико по $\epsilon$. Это означает, что пределы $\delta \rightarrow \infty$ и $\epsilon \rightarrow 0$ нельзя переставлять.

\section{3. $d$-МЕРНЫЙ ОПЕРАТОР ШРЕДИНГЕРА}

Подстановкой $\phi(r)=r^{-\nu} \psi(r)$, где $\nu=(d-2) / 2$, уравнение (7) сводится к уравнению Бесселя с потенциалом $u(r)$

$$
\psi_{r r}+\frac{1}{r} \psi_{r}+\left(k^{2}-\frac{\nu^{2}}{r^{2}}\right) \psi+\epsilon u(r) \psi=0 .
$$

При $\epsilon=0$ сушествуют два независимых решения: $\psi=J_{\nu}(k r)$ и $\psi=N_{\nu}(k r)$, оба решения осциллируют на бесконечности (см. [11, формула (8.451)]). Будем строить решение уравнения (23) при $k=i \kappa(\epsilon)$ и $0<\epsilon \ll 1$ в виде разложения Фурье-Бесселя

$$
\psi=\int_{0}^{\infty} k \alpha(k) J_{\nu}(k r) d k
$$

Используя формулу ортогональности

$$
k \int_{0}^{\infty} r J_{\nu}(k r) J_{\nu}\left(k^{\prime} r\right) d r=\delta\left(k-k^{\prime}\right),
$$

сводим уравнение (23) к интегральному уравнению

$$
\left(k^{2}+\kappa^{2}\right) \alpha(k)=\epsilon \int_{0}^{\infty} k^{\prime} K\left(k, k^{\prime}\right) \alpha\left(k^{\prime}\right) d k^{\prime},
$$


где

$$
K\left(k, k^{\prime}\right)=\int_{0}^{\infty} r u(r) J_{\nu}(k r) J_{\nu}\left(k^{\prime} r\right) d r .
$$

Асимптотика функции Бесселя $J_{\nu}(z)$ при $z \rightarrow 0$ имеет вид

$$
J_{\nu}(z) \rightarrow \frac{z^{\nu}}{2^{\nu} \Gamma(1+\nu)}
$$

(см. [11, формула (8.440)]). Исследуем поведение уравнения $(25)$ при $k, k^{\prime} \rightarrow 0$, выделяя масштабные множители:

$$
\alpha(k)=\frac{k^{\nu} \beta(k)}{k^{2}+\kappa^{2}}, \quad K\left(k, k^{\prime}\right)=k^{\nu} k^{\prime \nu} \Pi\left(k, k^{\prime}\right) .
$$

При $\kappa(\epsilon) \rightarrow 0$ интеграл в правой части $(25)$ с учетом $(26)$ оказьвается сингулярным при $k^{\prime} \rightarrow 0$, если $\nu \leqslant 0$ или эквивалентно $d \leqslant 2$. В этом случае при $\epsilon \rightarrow 0$ самосогласованное асимптотическое решение для нового связанного состояния сушествует. Полагая $\kappa(\epsilon)>0$, преобразуем уравнения $(25)$ и $(26)$ при $0<d<2$ к виду

$$
\beta(k)=\frac{\epsilon \kappa^{d-2} \pi}{2 \sin \left(\pi \frac{d}{2}\right)} \Pi(k, 0) \beta(0)+\epsilon \int_{0}^{\infty} \frac{k^{\prime d-1} d k^{\prime}}{k^{\prime 2}+\kappa^{2}}\left[\Pi\left(k, k^{\prime}\right) \beta\left(k^{\prime}\right)-\Pi(k, 0) \beta(0)\right],
$$

при этом мы воспользовались формулой (3.241) из [11]:

$$
\int_{0}^{\infty} \frac{z^{d-1} d z}{1+z^{2}}=\frac{\pi}{2 \sin \left(\pi \frac{d}{2}\right)}, \quad 0<d<2
$$

Из уравнения (27) следует, что асимптотический баланс достигается при $\epsilon \kappa^{d-2} \sim O(1)$, поэтому мы полагаем $\kappa^{2-d}=\epsilon \kappa_{1}+\epsilon^{2} \kappa_{2}+O\left(\epsilon^{3}\right)$. Условия совместности уравнения (27) приводят к следуюшим выражениям для $\kappa_{1}, \kappa_{2}$ :

$$
\begin{aligned}
& \kappa_{1}=\frac{\Gamma\left(1-\frac{d}{2}\right)}{2^{d-1} \Gamma\left(\frac{d}{2}\right)} N_{d}, \\
& \kappa_{2}=\frac{\Gamma\left(1-\frac{d}{2}\right)}{2^{d-1} \Gamma\left(\frac{d}{2}\right)} \iint_{0}^{\infty} r^{\frac{d}{2}} R^{\frac{d}{2}} u(r) u(R) Q(r, R) d r d R,
\end{aligned}
$$

где

$$
\begin{gathered}
N_{d}=\int_{0}^{\infty} r^{d-1} u(r) d r, \\
Q(r, R)=\lim _{\kappa \rightarrow 0} \int_{0}^{\infty} \frac{k d k}{k^{2}+\kappa^{2}}\left[J_{\nu}(k r) J_{\nu}(k R)-\frac{k^{2 \nu} r^{\nu} R^{\nu}}{2^{2 \nu}[\Gamma(1+\nu)]^{2}}\right],
\end{gathered}
$$

при этом мы использовали соотношение

$$
\Gamma(x) \Gamma(1-x)=\frac{\pi}{\sin (\pi x)} .
$$


Необходимым условием положительности параметра $\kappa(\epsilon)$ является условие $N_{d}>0$ (т.е. $\left.\kappa_{1}>0\right)$. Чтобы проверить конечность коэффициента $\kappa_{2}$, мы вычислим его явно. Воспользовавшись формулами $(6.577),(8.445)$ и $(8.485)$ из $[11]$, находим для $Q(r, R)$ при $R>r$

$$
Q(r, R)=\lim _{\kappa \rightarrow 0^{+}}\left[I_{\nu}(\kappa r) K_{\nu}(\kappa R)-\frac{\kappa^{2 \nu} r^{\nu} R^{\nu} \pi}{2^{2 \nu+1}[\Gamma(1+\nu)]^{2} \sin [\pi(1+\nu)]}\right]=\frac{r^{\nu}}{2 \nu R^{\nu}}
$$

Теперь выражение для $\kappa_{2}$ можно переписать в виде

$$
\begin{aligned}
\kappa_{2}= & -\frac{\Gamma\left(1-\frac{d}{2}\right)}{(2-d) 2^{d-1} \Gamma\left(\frac{d}{2}\right)} \iint_{0}^{\infty} r^{d-1} R^{d-1} u(r) u(R) \times \\
& \times\left[r^{2-d} \Theta(r-R)+R^{2-d} \Theta(R-r)\right] d r d R .
\end{aligned}
$$

Асимптотика нового собственного значения задачи (7) при $0<d<2$ имеет вид

$$
\kappa(\epsilon)=\epsilon^{\frac{1}{2-d}}\left(\frac{\Gamma\left(1-\frac{d}{2}\right)}{2^{d-1} \Gamma\left(\frac{d}{2}\right)} N_{d}\right)^{\frac{1}{2-d}}[1+O(\epsilon)]
$$

где $N_{d}>0$. В пределе $d \rightarrow 1$ формулы (28) и (31) переходят в формулы (13) и (15) при $\delta \equiv 1$, если потенциал симметричен, $u(-x)=u(x)$. Чтобы доказать это утверждение, воспользуемся тождеством

$$
r \Theta(r-R)+R \Theta(R-r)=\frac{1}{2}(|r-R|+|r+R|), \quad r, R \geqslant 0 .
$$

В пределе $d \rightarrow 2^{-}$коэффициенты $\kappa_{1}, \kappa_{2}$ и высших членов асимптотического разложения расходятся, так что все члены асимптотического разложения для $\kappa(\epsilon)$ становятся сравнимыми. В качестве предварительной оценки возьмем $d=2-\epsilon \mu$ и рассмотрим предел $\mu \rightarrow 0^{+}$в выражениях (28) и (31):

$$
\kappa^{\epsilon \mu}=\frac{N_{2}}{\mu}-\frac{N_{2}^{2}}{\mu^{2}}+O\left(\mu^{-3}, \epsilon\right) .
$$

Логарифмируя это выражение, получаем оценку предела $\mu \rightarrow 0^{+}$, выходящую за рамки степенного асимптотического разложения,

$$
\epsilon \ln \kappa=\lim _{\mu \rightarrow 0^{+}} \frac{1}{\mu} \ln \left(\frac{N_{2}}{\mu}-\frac{N_{2}^{2}}{\mu^{2}}+\cdots\right) \approx \lim _{\mu \rightarrow 0^{+}} \frac{1}{\mu} \ln \left(\frac{N_{2}}{N_{2}+\mu}\right)=-\frac{1}{N_{2}} .
$$

Поскольку в случае $d=2$ эта оценка выходит за рамки степенного разложения, мы получим асимптотику прямым методом. Для этого мы преобразуем интегральное уравнение (25) с учетом представления (26) к виду

$$
\beta(k)=-\epsilon \ln \kappa K(k, 0) \beta(0)-\frac{\epsilon}{2} \int_{0}^{\infty} d k^{\prime} \ln \left(k^{\prime}+\kappa^{2}\right) \frac{\partial}{\partial k^{\prime}}\left[K\left(k, k^{\prime}\right) \beta\left(k^{\prime}\right)\right] .
$$


Уравнение (32) удовлетворяется в ведушем порядке, если справедливо следуюшее асимптотическое разложение: $\ln \kappa(\epsilon)=-\epsilon^{-1} \kappa_{-1}+\kappa_{0}+O(\epsilon)$, где $\kappa_{-1}>0$. Коэффициенты $\kappa_{-1}$ и $\kappa_{0}$ имеют вид

$$
\begin{aligned}
\kappa_{-1} & =\frac{1}{N_{2}} \\
\kappa_{0} & =\frac{1}{N_{2}^{2}} \iint_{0}^{\infty} r R u(r) u(R) Q(r, R) d r d R,
\end{aligned}
$$

где

$$
Q(r, R)=\lim _{\kappa \rightarrow 0^{+}}\left[\int_{0}^{1} \frac{k d k}{k^{2}+\kappa^{2}}\left(J_{0}(k r) J_{0}(k R)-1\right)+\int_{1}^{\infty} \frac{k d k}{k^{2}+\kappa^{2}} J_{0}(k r) J_{0}(k R)\right] .
$$

Используя те же вычисления, что и в (30), и формулу (8.446) из [11], получаем для $\kappa_{0}$ явное выражение

$$
\kappa_{0}=-\gamma+\ln 2-\frac{1}{N_{2}^{2}} \iint_{0}^{\infty} r R u(r) u(R)[(\ln r) \Theta(r-R)+(\ln R) \Theta(R-r)] d r d R
$$

Эта формула, хотя и отличается по виду от формулы (35) Апенко [5], фактически ей эквивалентна. Апенко получил эту формулу с помошью предельного перехода $d \rightarrow 2^{+}$. Мы провели анализ уравнения (7) непосредственно при $d=2$ и нашли ту же асимптотику нового собственного значения:

$$
\kappa(\epsilon)=c \exp \left(-\epsilon^{-1} N^{-1}\right)[1+O(\epsilon)]
$$

где $c=\exp \left(\kappa_{0}\right)$ и $N_{2}>0$.

Наконец, упомянем тот факт, что выражения (33) и (35) допускают обобщения [5]

$$
\begin{gathered}
\kappa_{-1}=\frac{2 \pi}{M}, \quad M=\int_{\mathcal{R}^{2}} u(\mathbf{r}) d \mathbf{r} \\
\kappa_{0}=-\gamma+\ln 2-\frac{1}{M^{2}} \int_{\mathcal{R}^{2}} \int_{\mathcal{R}^{2}} u(\mathbf{r}) u(\mathbf{R}) \ln |\mathbf{r}-\mathbf{R}| d \mathbf{r} d \mathbf{R} .
\end{gathered}
$$

Если потенциал радиально-симметричен, $u(\mathbf{r})=u(r)$, выражения (36) и (37) сводятся соответственно к (33) и (35) с помошью формул $M=2 \pi N_{2}$ и

$$
\frac{1}{2 \pi} \int_{0}^{2 \pi} \ln \left(1+a^{2}-2 a \cos \theta\right) d \theta=0, \quad 0 \leqslant a<1
$$

Последняя формула следует из теоремы о среднем значении для гармонической функции $\ln \left(x^{2}+y^{2}\right)$ в двумерном пространстве (см. также [11, формула (4.224)]). Выражения (36) и (37) можно получить в спектральной задаче для двумерного оператора Шредингера с произвольным потенциалом $u(\mathbf{r})$ с помошью двойного разложения Фурье. 


\section{4. ЗАКЛЮЧЕНИЕ}

Мы получили асимптотики новых собственных значений линейных задач (2) и (7) в пределе малого потенциала. Наши результаты воспроизводят частные предельные случаи, рассмотренные в работах $[3-5,8]$. Главные особенности нашего подхода следующие.

Данный метод можно использовать в линейных задачах с асимптотически постоянным потенциалом, чтобы определить, существует ли пороговое значение амплитуды потенциала, при котором появляется новое собственное значение. Порог сушествует, если интегральное представление решения, возникаюшее при использовании фурье-разложения, не имеет сингулярных членов при $\epsilon \rightarrow 0$. В противном случае порог отсутствует и асимптотический анализ позволяет находить аналитическую зависимость нового собственного значения от $\epsilon$.

С помошью этого метода можно находить для нового собственного значения не только члены ведущего порядка, но и поправки следующего порядка, что полезно при оценке сходимости асимптотического ряда. В задачах, где новое собственное значение экспоненциально мало, наш метод позволяет находить предэкспоненциальный фактор. Наконец, с помощью интегрального представления для поправки первого порядка можно анализировать задачи, где члены ведушего порядка обрашаются в нуль (см. [3]).

Благодарности. Мы признательны А. Брадни, Ф. Калоджеро, А. Фокасу и И. М. Сигалу за полезные обсуждения. Д.П. благодарит комитет НАТО за финансовую поддержку, предоставленную NSERC; K. С. благодарит NSERC за финансовую поддержку (грант OGP0046179).

\section{Список литературы}

[1] F. Calogero, A. Degasperis. Spectral Transform and Solitons. Amsterdam: North-Holland Publishing Company, 1982.

[2] Л. Д. Ландау, Е. М. Лифииц. Курс теоретической физики. Т. 3. Квантовая механика. Нерелятивистская теория. М.: Наука, 1974.

[3] B. Simon. Ann. Phys. (N. Y. ). 1976. V. 97. P. 279-288.

[4] M. Klaus, B. Simon. Ann. Phys. (N. Y. ). 1980. V. 130. P. 251-281.

[5] S. M. Apenko. J. Phys. A. 1998. V. 31. P. 1553-1562.

[6] Yu. S. Kivshar, D. E. Pelinovsky, T. Cretegny, M. Peyrard. Phys. Rev. Lett. 1998. V. 80. P. 5031-5035.

[7] I. V. Barashenkov, D. E. Pelinovsky, E. V. Zemlyanaya. Phys. Rev. Lett. 1998. V. 80. P. 5117-5120.

[8] D. E. Pelinovsky, C. Sulem. J. Math. Phys. 1998. V. 39. P. 6552-6572.

[9] Y. Kodama, M. J. Ablowitz, J. Satsuma. J. Math. Phys. 1982. V. 23. P. 564-576.

[10] A. A. Minzoni, T. Miloh. Wave Motion. 1994. V. 20. P. 131-142.

[11] И. С. Градштейн, И. М. Рыжик. Таблицы интегралов, сумм, рядов и произведений. М.: Физматгиз, 1971. 